\title{
A NOTE ON THE VARIANCE OF THE NUMBER OF LOCI HAVING A GIVEN GENE FREQUENCY
}

\author{
W. J. EWENS \\ Department of Biology, University of Pennsylvania, Philadelphia, Pennsylvania 19174 \\ and \\ T. MARUYAMA
}

National Institute of Genetics, Mishima, Japan 411

Manuscript received May 20, 1974

Revised copy received November 7, 1974

\begin{abstract}
In a recent note in this journal (MARUYaMa 1973), one of us has considered, for a certain genetic model, the variance of the distribution of the number of loci having a given gene frequency. The formula given is incorrect. This brief note considers this problem and notes the correct solution in one particular case.
\end{abstract}

\section{Variance of the Number of Loci}

CONSIDER a diploid population of fixed size $N$; each individual in the population possesses an infinite sequence of gene loci. Mutations occur at average rate $u$ per gamete per generation according to the following scheme: a mutation always occurs at a homoallelic $\left(A_{1} A_{1}\right)$ locus. Once the mutation has occurred, no further mutation occurs at this locus. Thus segregation continues at this locus until either the original allele $A_{1}$ or the mutant $A_{2}$ is lost from the population. The number of generations required for such loss is a random variable, as is the number of generations that the frequency $x$ of $A_{1}$ remains in any arbitrary interval during the segregation progess.

Let $T$ be the (random) time that the frequency of $A_{1}$ remains in some small interval $(x-1 / 2 \Delta, x+1 / 2 \Delta)$ before loss of fixation of $A_{1}$. Further, in any generation, let $m$ be the (random) number of loci at which the frequency of the $A_{1}$ allele is in this interval. Assuming the same fitness parameters at all loci, we have by standard ergodic arguments

$$
E(m)=2 N u E(T) .
$$

This formula, with an explicit expression for $E(T)$ (EwENs 1963, 1964; KimUra 1964) that will depend on the assumption made concerning the model describing the process of gene frequency change at any site, is equation (1) in MARUYaMa (1973). 
To obtain the variance of $m$, it is necessary to make some assumption about the variance of the distribution of the number of new mutants per generation. For a deterministic input mutation process, MaruYama then claimed that

$$
E\left(m^{2}\right)=2 N u E\left(T^{2}\right),
$$

and employing a known expression for $E\left(T^{2}\right)$ for the gene frequency change model assumed, used (2) to obtain an expression for $E\left(m^{2}\right)$. However, there is no theorem in probability theory asserting the truth of (2). Indeed (2) is incorrect, as can be seen by noting that the dimension of the right-hand side is $(\text { time })^{-1} \times(\text { time })^{2}=($ time $)$, whereas the left-hand side is a dimensionless quantity. It follows that any argument relying on (2), and in particular much of the argument in MARUYAMA (1973), is invalid.

The problem of what formulae should be used for the quantities in question is in fact extremely difficult. The only case in which significant simplifications are possible is for a Poisson input mutation, which is possibly the most realistic assumption in real-world terms. If we ignore linkage between sites and assume the various gene frequency change processes are independent, then under the Poisson assumption the number of loci in any generation for which the frequency of $A_{1}$ is in $(x-1 / 2 \Delta, x+1 / 2 \Delta)$ is easily shown to be Poisson random variable, with mean given by (1) above. In this case, of course, the variance will be identical to the mean, and is thus also given by (1).

\section{LITERATURE CITED}

Ewens, W. J., 1963 The diffusion equation and a pseudo-distribution in genetics. J. Roy. Stat. Sac. B, 25: 405-412. - - 1964. The pseudo-transient distribution and its uses in genetics. J. Appl. Prob. 1 : 141-156.

Kimura, M., 1964 Diffusion models in population genetics. J. Appl. Prob. 1 : 177-232.

Maruyama, T., 1973 The variance of the number of loci having a given gene frequency. Genetics 73: $361-366$. 